\title{
Über die Constitution des Andalusits und des Disthens
}

von

\author{
Prof. Karl Zulkowski.
}

Aus dem chemisch-technologischen Laboratorium der k. k. deutschen technischen Hochschule in Prag.

(Vorgelegt in der Sitzung am 25. October 1900.)

Ich habe vor einiger Zeit mit mehreren glasbildenden Säuren, wie z. B. der Kieselsäure, Borsäure, Thonerde, Zinnsäure Versuche angestellt, wie sich dieselben gegen schmelzende Alkalicarbonate verhalten, und hiebei gefunden, dass jede derselben eine ausgesprochene Neigung zeigt, Metaverbindungen zu bilden; selbst dann, wenn man diesen Säuren einen größeren Alkaliüberschuss darbietet und somit der Entstehung von Orthoverbindungen Vorschub leistet.

Auf das hin war es mir möglich, auf die Constitution der Gläser, der hydraulischen Bindemittel und der Schlacken näher einzugehen und ein ziemlich dunkles Gebiet der reinen und angewandten Chemie aufzuhellen und späteren Forschungen zugänglicher zu machen. ${ }^{1}$

Die von mir ausgeübte Untersuchungsmethode gestattet nicht nur gewisse Kunstproducte, sondern auch viele natürlich vorkommende Mineralien einer rationellen Analyse zu unterwerfen, um eine Vorstellung über deren chemische Constitution zu gewinnen. So war es mir möglich, endgiltig festzustellen, dass dem reinen Kaolin die Constitutionsformel

1 Siehe die Zeitschrift $\gg$ Chemische Industrie , Jahrg. 1899, S. 280, Jahrg. 1900, S. 108 und 346. 


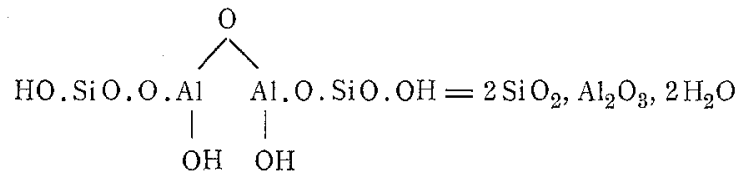

zukommen müsse, weil derselbe, selbst mit einem großen Überschusse von Alkalicarbonat niedergeschmolzen, nur 2 Molecüle Kohlendioxyd austreibt, somit nur 2 Molecüle Alkali bindet.

Bei diesem Schmelzprocesse bleibt der ganze Atomcomplex erhalten, und es entsteht offenbar nachstehende Verbindung:

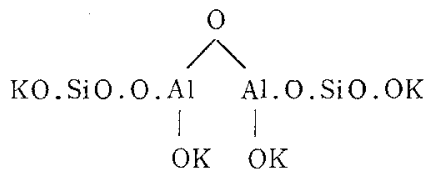

Ich habe bei dieser Gelegenheit auch dargethan, dass die Thonerde die bereits vermuthete Eigenschaft besitzt, Verbindungen mit der Kieselsäure einzugehen, die nicht als Salze im gewöhnlichen Sinne des Wortes gelten können, sondern als Aluminiumkieselsäuren aufzufassen sind, und dass letztere in gewissen thonerdehaltigen Hochofenschlacken und Gläsern vorkommen müssen.

Diese Ergebnisse bewogen mich, zwei isomere Thonerdesilicate, nämlich den Andalusit und Disthen zu untersuchen, deren Zusammensetzung der empirischen Formel $\mathrm{SiO}_{2}, \mathrm{Al}_{2} \mathrm{O}_{3}$ entspricht.

Groth ist der Ansicht, dass der Unterschied in ihrer Constitution zurückzuführen sei auf die Verschiedenheit der beiden mit der Thonerde verbundenen Kieselsäuren. Nach ihm wäre der Andalusit ein Orthosilicat, welchem derselbe die Formel

$$
\text { Sik } \begin{aligned}
& 0 \\
& 0 \\
& 0 \\
& O . A l O
\end{aligned}
$$

ertheilt. Diese Formel ist jedoch sehr wenig wahrscheinlich, weil die beiden Aluminiumatome unter sich ohne Bindung sind, und es müsste die Formel entsprechend modificiert werden, 
wenn man dem Andalusit die Structur eines Orthosilicates ertheilen wollte. Diese kann nur folgende sein:

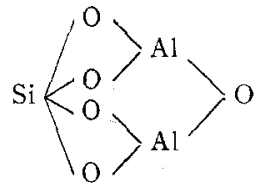

Für den Disthen oder Cyanit nimmt Groth die Constitution eines Metasilicates an, dem er die Formel<smiles>O1[Al]2O[Al]3O[Al]1O[Al]3O2</smiles>

ertheilt, und erklärt dadurch auch die schwierigere Zersetzbarkeit dieses Minerals, weil bekanntlich Metasilicate schwerer zersetzbar sind. ${ }^{1}$

Nach meinen Erfahrungen, die ich beim Schmelzen der verschiedenen glasbildenden Säurenanhydride mit Pottasche machte, bilden sich dabei immer die Kaliverbindungen gewisser Pyrosäuren von geringerem Sättigungsvermögen als die der entsprechenden Orthosäuren; so z. B. lieferten

$$
\begin{aligned}
& \mathrm{SiO}_{2} \quad \mathrm{SiO}(\mathrm{OK})_{2} \\
& \text { B. OK } \\
& \mathrm{B}_{2} \mathrm{O}_{3} \\
& \text { O } \\
& >_{\mathrm{B}, \mathrm{OK}}^{\mathrm{O}} \\
& \mathrm{Al}_{2} \mathrm{O}_{3} \\
& \text { Al.OK } \\
& 0<>_{\mathrm{A} 1.0 \mathrm{~K}}^{\mathrm{O}}
\end{aligned}
$$

Daraus würde sich also ergeben, dass beim Schmeizen des Andalusits mit überschüssiger Pottasche eine Umlagerung zu einem Metasilicate erfolgen würde, in welchem die Thonerde in der Form des Diaspor, also zweiwertig auftritt, wie dies aus folgender Gleichung hervorgeht:

1 Siehe chemische Mineralogie von Dr. Reinhard Brauns, S. 439. 


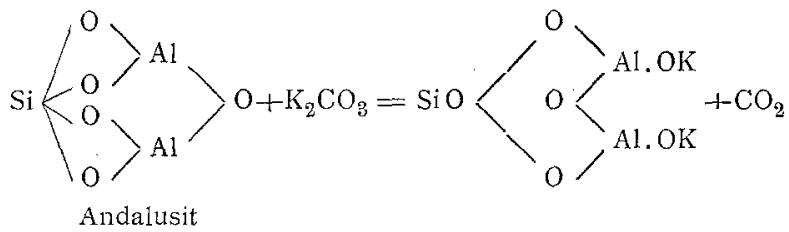

Viel einfacher, d. h. ohne innerer Umlagerung, würde der Schmelzprocess des Disthens verlaufen, an dessen Thonerderest sich das Alkali in gleicher Weise anlagern müsste, um dieselbe Verbindung in nachstehender Weise zu bilden:

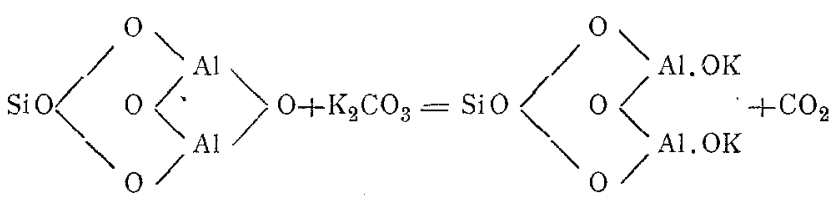

Disthen

In beiden Fällen müssten für je 1 Molecül Andalusit oder Disthen 1 Molecül Kohlendioxyd ausgetrieben werden. Um hierüber Klarheit zu erlangen, habe ich meine beiden Schüler Emil Kraus und Alberto Goetzl beauftragt, diese zwei Mineralien solchen Schmelzversuchen zu unterwerfen, wie ich dies seinerzeit mit Thonerde und Kaolin gethan habe.

Zu diesem Behufe wurde ungefähr $1 g$ des feingepulverten und getrockneten Minerals mit nahezu 3 Molecülen entwässerter Pottasche in einem Platintiegel innigst gemischt und auf dem Gebläse so lange geschmolzen, bis der durch die ausgetriebene Kohlensäure entstandene Gewichtsverlust nahezu constant blieb. Zu dessen Erkennung wurde die Schmelzung von Zeit zu Zeit unterbrochen und der erkaitete Tiegel gewogen. Die Pottasche ist wegen der viel geringeren Flüchtigkeit in der Schmelztemperatur der Soda vorzuziehen. Die große Menge dieses Schmelzmittels, nämlich 3 Molecüle auf 1 Molecül Mineral ist durchaus nothwendig, weil die entstandene Alkaliverbindung schwer schmelzbar ist und eine vollständige Verbindung nur dann erfolgt, wenn die Schmelze durch die überschüssige Pottasche mindestens eine breiige Beschaffenheit erhält.

Dass trotz dieses großen Überschusses der ganze Atomcomplex erhalten bleibt, ist ein Beweis, dass diese zwei Mine- 
ralien nicht den Charakter eines Salzes besitzen, sondern die Anhydride von zwei Aluminiumkieselsäuren darstellen.

Bei der Annahme, dass auf 1 Molecül $\mathrm{SiO}_{2}, \mathrm{Al}_{2} \mathrm{O}_{3}=162.6 \mathrm{~g}$ 1 Molecül $\mathrm{K}_{2} \mathrm{CO}_{3}$ reagiere, also 1 Molecül $\mathrm{CO}_{2}=44 \mathrm{~g}$ entweichen sollte, würde je $1 \mathrm{~g}$ Mineral einen Schmelzverlust von $0 \cdot 2706 \mathrm{~g}$ ergeben.

Die von Herrn Emil Kraus mit dem Andalusit durchgeführten drei Schmelzversuche haben die Groth'sche Annahme nicht bestätigt, wie dies in nachfolgender Zusammenstellung ersichtlich ist.

I. Versuch.

Einwage von Mineral $1.0072 \mathrm{~g}$.

Einwage von Pottasche $2 \cdot 578 \mathrm{~g}$.

Der theoretische Gewichtsverlust beträgt $0.2725 \mathrm{~g}$.

\begin{tabular}{|c|c|c|}
\hline $\begin{array}{c}\text { Schmelzzeit } \\
\text { in Stunden }\end{array}$ & $\begin{array}{c}\text { Gewichtsverlust } \\
\text { in Gramm }\end{array}$ & $\begin{array}{c}\text { Molecuile Alkali, } \\
\text { gebunden }\end{array}$ \\
\hline $1 / 2$ & 0.4124 & 1.50 \\
1 & 0.4210 & 1.54 \\
\hline
\end{tabular}

II. Versuch.

Einwage an Mineral $0.8889 \mathrm{~g}$.

Einwage an Pottasche $2 \cdot 5536 \mathrm{~g}$.

Der theoretische Schmelzverlust beträgt $0 \cdot 2407 \mathrm{~g}$.

\begin{tabular}{|c|c|c|}
\hline $\begin{array}{c}\text { Schmelzzeit } \\
\text { in Stunden }\end{array}$ & $\begin{array}{c}\text { Gewichtsverlust } \\
\text { in Gramm }\end{array}$ & $\begin{array}{c}\text { Molecuile Alkali, } \\
\text { gebunden }\end{array}$ \\
\hline \hline $1 / 2$ & $0 \cdot 1901$ & 0.76 \\
1 & 0.3368 & $1 \cdot 39$ \\
$11 / 2$ & 0.3515 & $1 \cdot 46$ \\
$11^{3 / 4}$ & 0.3649 & $1 \cdot 51$ \\
2 & 0.3652 & 1.52 \\
\hline
\end{tabular}


III. Versuch.

Einwage an Mineral 1.0000 g. Einwage an Pottasche $2 \cdot 7730 \mathrm{~g}$.

Der theoretische Schmelzverlust beträgt $0 \cdot 2706 \mathrm{~g}$.

\begin{tabular}{|c|c|c|}
\hline $\begin{array}{c}\text { Schmelzzeit } \\
\text { in Stunden }\end{array}$ & $\begin{array}{c}\text { Gewichtsverlust } \\
\text { in Gramm }\end{array}$ & $\begin{array}{c}\text { Molecuile Alkali, } \\
\text { gebunden }\end{array}$ \\
\hline \hline $1 / 2$ & 0.3449 & 1.27 \\
1 & 0.4081 & 1.50 \\
$11 / 4$ & 0.4159 & 1.53 \\
$11 / 2$ & 0.4167 & 1.54 \\
\hline
\end{tabular}

Alle drei Schmelzversuche ergaben somit in übereinstimmender Weise einen Gewichtsverlust, der fast genau $1 \frac{1}{2}$ mal so groß ist als derjenige, welcher 1 Molecül $\mathrm{CO}_{2}$ oder 1 Molecül gebundenes Kali entsprechen würde; somit ist das Molecül jenes Aluminiumkieselsäureanhydrids, welches als Andalusit bezeichnet wird, doppelt so groß, als es die empirische Formel $\mathrm{SiO}_{2}, \mathrm{Al}_{2} \mathrm{O}_{3}$ verlangt. In gleicher Weise wurden von Alberto Goetzl zwei Schmelzversuche mit Disthen durchgeführt, deren Ergebnisse in nachfolgender Zusammenstellung verzeichnet sind.

I. Versuch.

Einwage an Mineral $1 g$.

Einwage an Pottasche $2 \cdot 59 \mathrm{~g}$.

Der theoretische Schmelzverlust beträgt $0.2706 g$.

\begin{tabular}{|c|c|c|}
\hline $\begin{array}{c}\text { Schmelzzeit } \\
\text { in Stunden }\end{array}$ & $\begin{array}{c}\text { Gewichtsverlust } \\
\text { in Gramm }\end{array}$ & $\begin{array}{c}\text { Molecüle Alkali, } \\
\text { gebunden }\end{array}$ \\
\hline \hline $1 / 2$ & 0.4010 & 1.48 \\
1 & 0.4184 & 1.50 \\
$11 / 4$ & 0.4185 & 1.54 \\
\hline
\end{tabular}


Einwage an Mineral $1 \mathrm{~g}$.

Einwage an Pottasche $2.59 \mathrm{~g}$.

Der theoretische Schmelzverlust beträgt $0 \cdot 2706 \mathrm{~g}$.

\begin{tabular}{|c|c|c|}
\hline $\begin{array}{c}\text { Schmelzzeit } \\
\text { in Stunden }\end{array}$ & $\begin{array}{c}\text { Gewichtsverlust } \\
\text { in Gramm }\end{array}$ & $\begin{array}{c}\text { Molecuile Alkali, } \\
\text { gebunden }\end{array}$ \\
\hline $1 / 4$ & 02725 & 0.99 \\
$1 / 2$ & 0.3458 & 1.27 \\
1 & 0.3986 & 1.46 \\
$11 / 4$ & 0.3998 & 1.47 \\
\hline
\end{tabular}

Ein dritter Versuch wurde von mir selbst durchgeführt und lieferte nachfolgende Resultate:

\section{Versuch.}

Einwage an Mineral $0.9947 \mathrm{~g}$.

Einwage an Pottasche $2 \cdot 5968 \mathrm{~g}$.

Der theoretische Schmelzverlust beträgt $0.2692 \mathrm{~g}$.

\begin{tabular}{|c|c|c|}
\hline $\begin{array}{c}\text { Schmelzzeit } \\
\text { in Stunden }\end{array}$ & $\begin{array}{c}\text { Gewichtsvertust } \\
\text { in Gramm }\end{array}$ & $\begin{array}{c}\text { Molecüle Alkali, } \\
\text { gebunden }\end{array}$ \\
\hline \hline $1 / 2$ & 0.3869 & 1.437 \\
$3 / 4$ & 0.4110 & 1.527 \\
1 & 0.4131 & 1.530 \\
\hline
\end{tabular}

Alle drei Versuche ergaben in übereinstimmender Weise, dass auf 1 Molecuil Disthen oder richtiger auf 1 Molecül der Verbindung $\mathrm{SiO}_{2}, \mathrm{Al}_{2} \mathrm{O}_{3}$ ebenfalls 1.5 Molecüle Kohlendioxyd kommen, somit muss das Molecül des Disthens ebenfalls doppelt so groß sein als angenommen wurde.

Diese überraschende Thatsache, welche auch hier die Ansicht von Groth nicht bestätigt, nöthigt zu der Annahme, dass die Isomerie beider Mineralien in der gegenseitigen Stellung 
der Silicium- und Aluminiumgruppen begründet ist. Für die Molecularformel $2 \mathrm{SiO}_{2}, 2 \mathrm{Al}_{2} \mathrm{O}_{3}$ kann die gegenseitige Stellung der Silicium- und Aluminiumgruppen eine dreifache sein; nämlich:
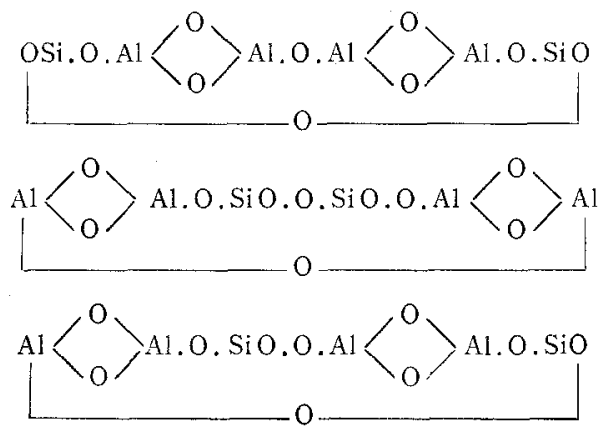

Es ist gewiss überraschend, dass es außer den vorhin genannten $z w e i$ Mineralien thatsächlich noch ein drittes, nämlich das als Sillimanit bezeichnete gibt, dessen chemische $\mathrm{Zu}$ sammensetzung ebenfalls der empirischen Formel $\mathrm{SiO}_{2}, \mathrm{Al}_{2} \mathrm{O}_{3}$ entspricht. ${ }^{1}$

Alle drei Formeln drücken die Anhydride dreier Aluminiumkieselsäuren aus, und die beim Schmelzen mit Pottasche entstehenden Verbindungen werden eine Zusammensetzung besitzen, welche durch nachstehende Formeln dargestellt werden kann:

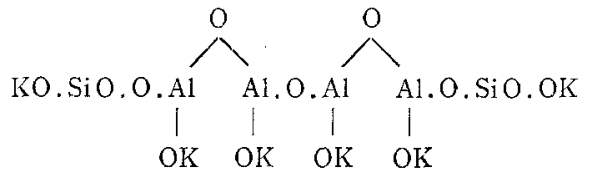

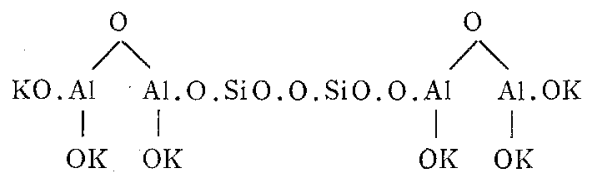

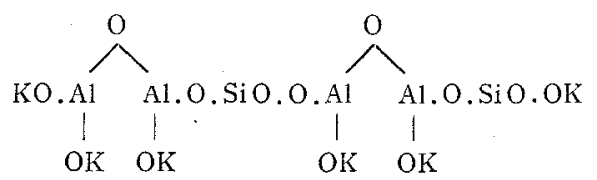

1 Ich behalte mir vor, dieses Mineral in der nächsten Zeit in derselben Richtung zu untersuchen. 
In der ersten und zweiten Formel sind die Silicium- und Aluminiumgruppen symmetrisch gelagert und haben entweder die End-oder die Mittelstellung; in der dritten ist die Lagerung unsymmetrisch oder abwechselnd.

Obzwar diese Untersuchung die Frage der chemischen Constitution beider Mineralien nicht völlig entschieden hat, und es noch immer zweifelhaft erscheint, welche der drei Formeln dem Andalusit und Disthen zukommt, so ist dieser Fall doch sehr lehrreich, weil man sieht, dass die Verschiedenheit von mineralischen Gebilden, ebenso wie bei organischen Verbindungen in der Verschiedenheit der Stellung gewisser Atomgruppen begründet sein kann. 